\title{
A case report on a patient with Wolff-Parkinson-White syndrome presenting for scoliosis surgery
}

\author{
Authors: Ee-Ling, Ng; Nanthini, Pillay; Suan-Ling, Lim \\ Department of Paediatric Anaesthesia, KK Women's and Children's Hospital, Singapore
}

\begin{abstract}
Background
Wolff-Parkinson-White syndrome (WPW) is a congenital syndrome where there is abnormal conductive tissue between the atria and ventricles, providing a pathway for re-entrant tachycardia. It carries an increased risk of malignant tachyarrhythmias and sudden cardiac death. Inappropriate treatment of this syndrome could rapidly lead to clinical deterioration. Unfamiliarity with this rare condition $(0.1-0.3 \%$ of population) and difficult resuscitation in the prone position could further worsen outcomes.
\end{abstract}

\section{Case report}

We report a 16 year-old girl with WPW and Down syndrome who underwent T4 to L1 scoliosis surgery. She was first symptomatic at 4 years old, and subsequently diagnosed with WPW syndrome with characteristic features on electrocardiogram. She was asymptomatic after the first episode, had no

electrophysiologic study or radioablation done, and was not on anti-arrhythmics. Her parents were counseled on the increased risk of tachyarrhythmias, possibly requiring cardiopulmonary resuscitation (CPR). Adenosine and procainamide were made readily available in pre-calculated doses for use intra-operatively. The resuscitation plan was discussed and coordinated with the surgical team. We instituted invasive haemodynamic monitoring post-induction and defibrillation pads were applied at standard positions on the anterior chest prior to prone positioning. General anaesthesia was induced and maintained with propofol targetcontrolled infusion. Excessive increases in sympathetic or vagal tone were prevented, by using adequate analgesia with remifentanil and dexmedetomidine. Surgery proceeded uneventfully. Post-operative analgesia was managed with morphine nurse-controlled analgesia in view of the patient's intellectual disability. Ketamine is a common analgesic used in our centre for patients post scoliosis surgery, but this was avoided due to its pro-arrhythmic properties. Our patient had an uneventful recovery in the paediatric intensive care unit.

\begin{abstract}
Discussion
Haemodynamic stability and efficacy of resuscitation were major concerns in this case. Potential significant intra-operative blood loss and pain could precipitate a haemodynamically significant tachyarrhythmia.
\end{abstract}

There have been case reports describing the peri-operative management of WPW, and ACC-AHA 2015 guidelines on the management of tachyarrhythmias in these patients. However, we were unable to find a succinct guide to intra-operative arrhythmias in WPW. Moreover, these resources also do not address the unique circumstance of our patient needing prolonged major surgery in the prone position, and regional anaesthesia is not feasible for scoliosis surgery. In addition, given the varied pathophysiology of re-entrant tachyarrhythmias in WPW, use of an unsuitable anti-arrhythmic agent could potentially accelerate clinical deterioration.

Prone positioning complicates resuscitation in spine surgery. Sternal support is crucial, and may have to be provided in the form of the surgical assistant's clenched fist held at the midthoracic area. Turning the patient supine for CPR may cause delays, and in many circumstances may not be feasible unstable spine, protruding spinal implants etc. Moreover, risk of infection from an open wound and risk of neurological injury is a major concern.

Unfamiliarity with WPW impacted the anaesthetic plan, a concern mirrored in a survey we conducted in our institution. Out of 20 survey responses, 95\% had anaesthetised fewer than 5 WPW patients, and $35 \%$ have had no prior experience. We thus crystallized information in the literature to formulate an easy reference guide, and find that it would be useful to share our experience.

\section{Learning points}

WPW poses a significant risk of adverse cardiac outcome. In a patient undergoing major surgery with limited physical access for resuscitation, conscientious preparation and a clear, succinct treatment algorithm could make the difference.

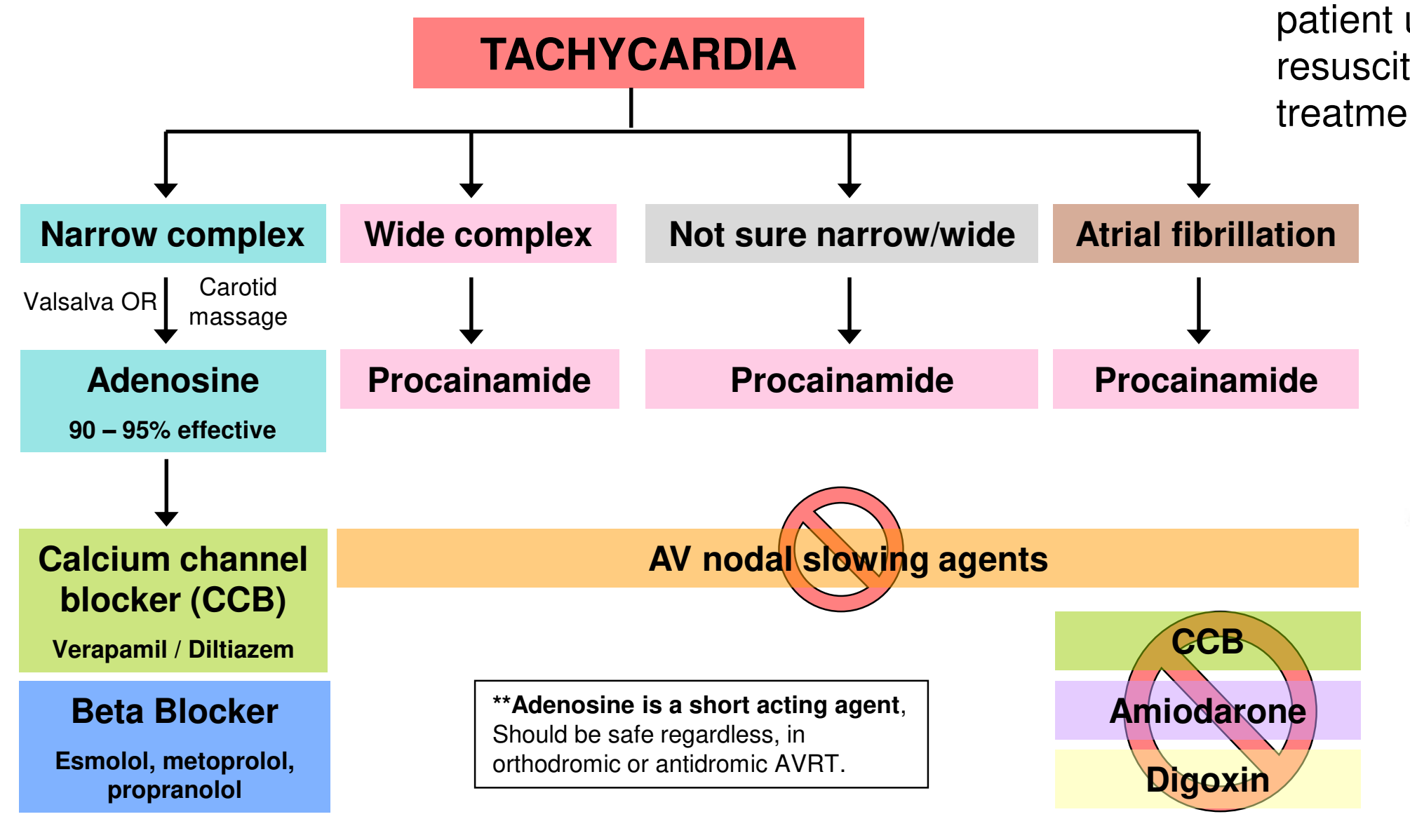

Unstable / refractory to meds $\rightarrow$ CARDIOVERT
CLINICAL PRESENTATION OF WPW (\%)

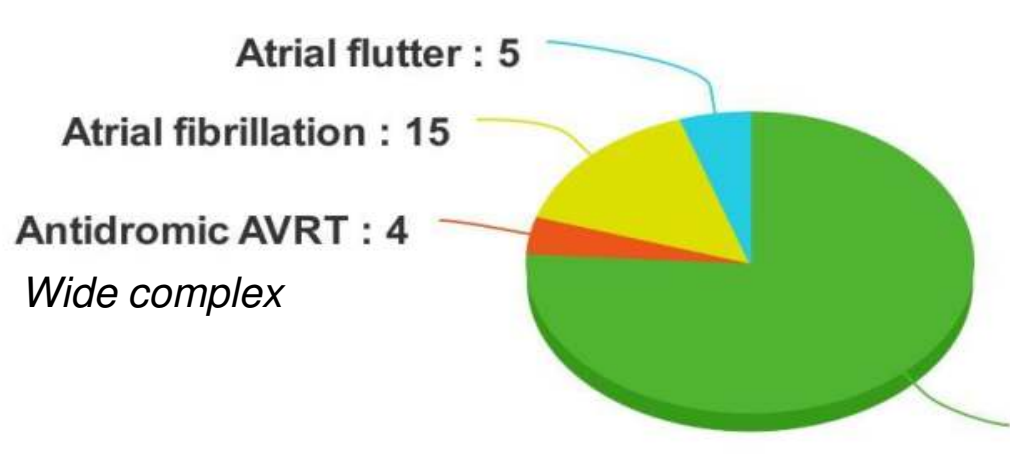

Atrial fibrillation/flutte Variable QRS - may be wide/narrow

Orthodromic AVRT : 76 Usually narrow complex May be wide complex if bundle branch block present

\section{References:}

1. Pappone C, Vicedomini G, Manguso F, et al. Wolff-Parkinson-White syndrome in the era of catheter ablation: insights from a registry study of 2169 patients. Circulation. 2014;130:811-9.

2. Bengali, Raheel et al. Perioperative Management of the Wolff-Parkinson-White Syndrome. Journal of

Cardiothoracic and Vascular Anesthesia , Volume 28 , Issue 5 , 1375-1386

3. Chung KY, Walsh TJ, Massie E. Wolff-Parkinson-White syndrome. Am Heart J. 1965; 69: 1-8.

4. 2015 ACC/AHA/HRS Guideline for the Management of Adult Patients with Supraventricular Tachycardia 